CFNUL 2/2000

\title{
Searching Scalar Resonances With Ultra-High ENERGY NEUTRINOS
}

\author{
L. Brücher ${ }^{a, 1}$, P. Keränen ${ }^{a, 2}$, J. Maalampi ${ }^{b, 3}$ \\ ${ }^{a}$ Centro de Física Nuclear de Universidade de Lisboa, \\ Av. Prof. Gama Pinto 2, 1649-003 Lisboa, Portugal \\ ${ }^{b}$ Department of Physics, Unversity of Helsinki \\ and \\ Helsinki Institute of Physics, \\ P.O. Box 9, FIN-00014 Helsinki, Finland
}

December 24, 2018

\begin{abstract}
We study the prospects of detecting signals of a resonant scattering of high-energy cosmic neutrinos on electrons in the atmosphere. Such a process is possible through an s-channel exchange of a isotriplet scalar particle predicted by some particle physics theories. We estimate the event rates for a reference detector setup with plausible assumptions on the interaction strengths and energy resolutions. We find as the most promising process the resonance production of tau neutrinos whose signature would be a "quiet" (in contrast with a hadronic "bang") production of the tau lepton followed by a more noisy decay in downstream.
\end{abstract}

PACS number(s): 12.60.Fr, 13.15.+g, 13.85.Tp, 14.80.Cp, 96.40.Pq, 96.40.Tv, 98.70.Sa

\footnotetext{
${ }^{1}$ e-mail: bruecher@cii.fc.ul.pt

${ }^{2}$ e-mail: keranen@cii.fc.ul.pt

${ }^{3}$ e-mail: Jukka.Maalampi@Helsinki.fi
} 


\section{Introduction}

It was suggested long ago by Glashow [1] that one could look for the effects of the $W$-boson in the spectrum of hadronic cascades produced via a resonant scattering of cosmic antineutrinos with electrons in the atmosphere. This suggestion was made before the discovery of the $W$-boson in laboratory experiments. We will revive Glashow's idea by applying it to another resonant process, the electron-neutrino scattering via an isotriplet scalar exchange. Isotriplet scalars are predicted by many models [2] and they may play role in the so-called seesaw mechanism of neutrino masses [3]. There is no evidence of their existence from laboratory experiments so far. On the other hand the data still allows their couplings to leptons to be large, in contrast with the couplings of the ordinary Higgs scalars 4 , 5. 5. We shall study whether the plans to build large neutrino telescopes and to use air shower arrays to detect horizontal neutrino-induced air showers would open a possibility to see signals of these particles in the cosmic ray spectra. We evaluate the event rates of the resonant isotriplet mediated electron-neutrino scattering for plausible isotriplet scalar masses in various detectors and compare them with the corresponding background rates.

We do not specify the model but assume simply that at the energies relevant for our studies there exists, in addition to the Standard Model particles, an isotriplet of scalar fields $\left(\Delta^{0}, \Delta^{-}, \Delta^{--}\right)$ carrying $S U(2)_{L} \times U(1)_{Y}$ quantum numbers $\left(I_{3}, Y\right)=(3,-2)$. The couplings of the triplet $\Delta$ to leptons are governed by the Lagrangian

$$
\mathcal{L}_{\Delta}^{\text {Yukawa }}=i h_{\ell \ell^{\prime}} \Psi_{\ell L}^{T} C \sigma_{2} \Delta \Psi_{\ell^{\prime} L}+\text { h.c. },
$$

where $\Psi_{\ell L}=\left(\nu_{\ell L}, \ell_{L}\right)$ and $\ell=e, \mu, \tau$. This interaction breaks the lepton number by two units. One should note that $\Delta$ does not couple to quarks, which has important consequences in respect to its possible astrophysical manifestations.

The interactions (ii) allow for the $e^{-} \nu_{e}$ annihilation in s-channel, whose astrophysical signal we will consider in the following. The present phenomenological constraints on the couplings $h_{\ell \ell^{\prime}}$ are the following (see e.g. 50):

$$
\begin{aligned}
h_{e \mu} h_{e e} & <3.2 \times 10^{-11} \mathrm{GeV}^{-2} \cdot M_{\Delta++}^{2} \\
h_{e e}^{2} & \lesssim 9.7 \times 10^{-6} \mathrm{GeV}^{-2} \cdot M_{\Delta^{++}}^{2} \\
h_{\mu \mu}^{2} & \lesssim 2.5 \cdot 10^{-5} \mathrm{GeV}^{-2} \cdot M_{\Delta^{++}}^{2} \\
h_{e e} h_{\mu \mu} & \lesssim 5.8 \cdot 10^{-5} \mathrm{GeV}^{-2} \cdot M_{\Delta^{++}}^{2} \\
h_{e \mu} h_{\mu \mu} & \lesssim 2 \cdot 10^{-10} \mathrm{GeV}^{-2} \cdot M_{\Delta^{++}}^{2}
\end{aligned}
$$

where $M_{\Delta^{++}}$is in $\mathrm{GeV}$. There exist no strict constraints on the corresponding couplings with $\tau$ leptons and neutrinos. It is conceivable to assume that the masses of the all members of the triplet $\Delta$ are more or less equal, and hence these limits can be taken to be valid also for the couplings of the singly charged triplet Higgs boson. In the following we will consider two scalar mass values, $150 \mathrm{GeV}$ and $300 \mathrm{GeV}$. In the former case the maximum values of the Yukawa couplings for the first two generations are $h_{e e}=0.47$ and $h_{\mu \mu}=0.75$. In the latter case the couplings can have any value up to the vacuum stability limit of $h_{\ell \ell^{\prime}}=\mathcal{O}(1)$.

We will consider the process

$$
\nu_{e} e^{-} \rightarrow \Delta^{-} \rightarrow \ell^{-} \nu_{\ell},(\ell=e, \mu, \tau) .
$$

The total cross section of the process in the laboratory frame is given by

$$
\sigma=\frac{h_{e e}^{2} h_{\ell \ell}^{2}}{4 \pi} \times \frac{m_{e} E_{\nu}}{\left[m_{\Delta^{-}}^{2}-2 m_{e} E_{\nu}\right]^{2}+\Gamma_{\Delta^{-}}^{2} m_{\Delta^{-}}^{2}},
$$

where $E_{\nu}$ is the energy of the cosmic neutrino and $\Gamma_{\Delta^{-}}$is the total width of the isotriplet scalar. One should emphasize that the cross section depends quadratically on $h_{e e}$ and $h_{\ell \ell}$. In our numerical 


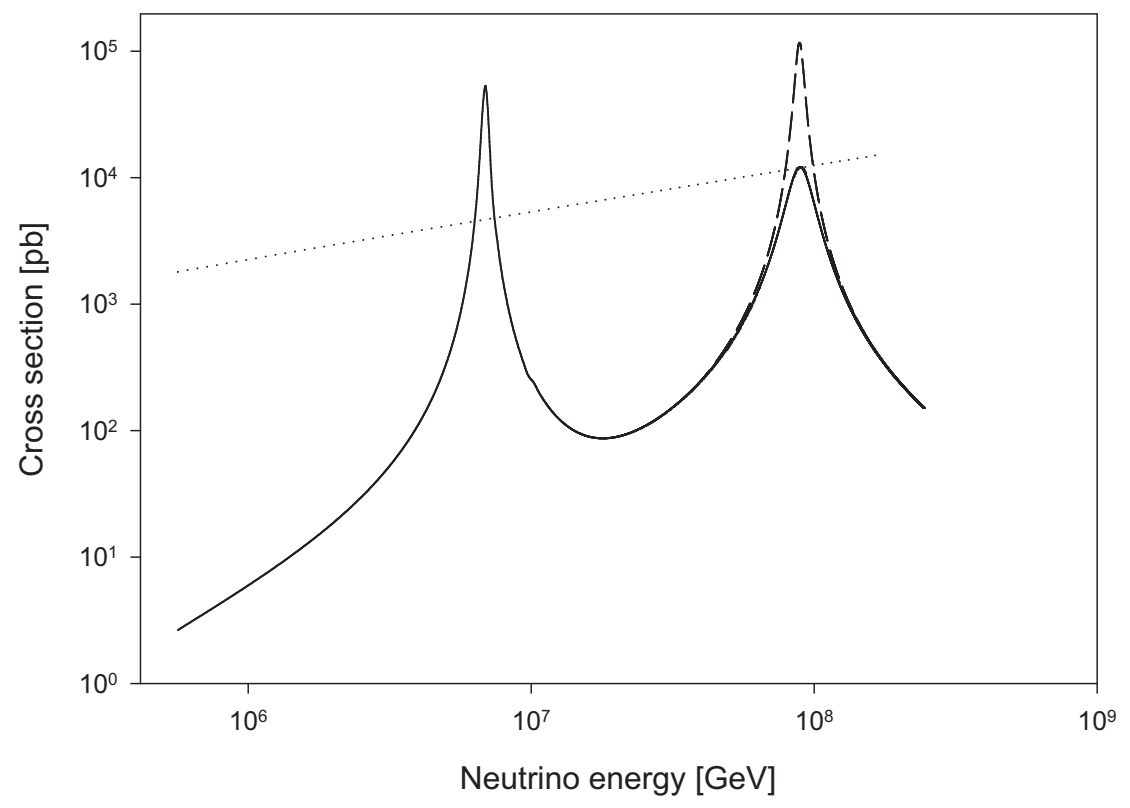

Figure 1: Glashow resonance (left peak) and $\Delta$-resonance (right peak) with $h_{e e}=h_{\ell \ell}=1$, $m_{\Delta}=300 \mathrm{GeV}$ and $\Gamma_{\Delta}=34.5 \mathrm{GeV}$ (solid line) and with $h_{e e}=1, h_{\mu}=h_{\tau}=0, m_{\Delta}=300 \mathrm{GeV}$ and $\Gamma_{\Delta}=11,5 \mathrm{GeV}$ (dashed line). The straight dotted line is the deep inelastic scattering cross section for the processes $\nu_{\ell} N \rightarrow \ell^{-} X$ and $\bar{\nu}_{\ell} N \rightarrow \ell^{+} X$.

calculations we assume for these coupling the largest values allowed by the present laboratory data. If their values in reality are less than these maximum values, the event rates presented below should be rescaled accordingly.

Fig. 1 represents the cross section of the process (3) for $m_{\Delta}=300 \mathrm{GeV}$ and $\Gamma_{\Delta}=34.5 \mathrm{GeV}$ (right peak) as a function of the cosmic neutrino energy, in comparison with the cross section of the Glashow process (left peak). In the same figure we also give the cross section for the deep inelastic $\nu_{\ell}$-nucleon and $\bar{\nu}_{\ell}$-nucleon scattering processes, which will build the main background in some detectors. Notice that for all processes only one neutrino (or antineutrino) flavour is produced; we assume that one can distinguish between different flavours. For neutrino energies between $10^{7} \mathrm{GeV} \lesssim E_{\nu} \lesssim 10^{12} \mathrm{GeV}$ this cross section is given by [6]

$$
\sigma_{\nu N+\bar{\nu} N}\left(E_{\nu}\right) \simeq 15.64 \times 10^{-36}\left(E_{\nu} / 10^{9} \mathrm{eV}\right)^{0.363} \mathrm{~cm}^{2}
$$

Note that the nucleon to electron ratio is around 2 for most materials, which will additionally increase this background.

Pion photoproduction processes in the sources $\left(p \gamma \rightarrow \pi^{+} n ; \pi^{+} \rightarrow \mu^{+} \nu_{\mu} ; \mu^{+} \rightarrow e^{+} \nu_{e} \bar{\nu}_{\mu}\right)$ suggest that no $\nu_{\tau}$ are produced in the sources, and $\nu_{\mu}$ are produced twice as many as $\nu_{e}$. The SuperKamiokande atmospheric neutrino data indicates that the muon neutrino has a large mixing with some other neutrino, presumably the tau neutrino [7]. The combined solar neutrino data favours large mixing angle solutions (LMA, LOW) for the electron neutrino mixing with an active neutrino [8]. This would lead the flavour composition of the extragalactic neutrino flux to be even in all flavours [9, 10], $N_{\tau}: N_{\mu}: N_{e}=1: 1: 1$. Note, however, that the discussion of the solar, atmospheric and laboratory neutrino data is not settled yet, both three and four neutrino solutions still being possible [11. Nevertheless, assuming the most plausible neutrino mixing scenario, all three charged leptons are produced in equal amounts in the deep inelastic scattering background processes described above.

Let us now move to the possible detection of the process (3). Currently two major detector designs for observing high energy neutrinos are discussed, namely atmospheric detectors and ice or water detectors. These detectors have in common that they detect fluorescent light emitted 
by energetic charged particles. Obviously, in our case the charged particle to be observed is the high energy lepton $\ell^{-}$produced in the $\Delta^{-}$decay. In case $\Delta^{-}$decays into $\nu_{e}$ and $e^{-}$, a short electromagnetic cascade will be produced, whose energy should be measurable. If the energy resolution of the detector is good enough, the $\Delta^{-}$-resonance should be distinguishable from the Glashow resonance. Therefore electrons are good candidates for detection. Unfortunately electrons and positrons are produced also in deep inelastic scattering processes that will form a serious background. A final state muon at these energies propagates a much longer distance than is the size of any imaginable detector, and furthermore the energy resolution for muons is much worse than for electrons. Thus they are not good candidates for the detection of the process (3).

In contrast, the tau lepton produced by $10^{8} \mathrm{GeV}$ neutrino will decay typically within $2-3 \mathrm{~km}$ from its production point producing a hadronic shower. The signal is different from the so-called "double bang event" produced by $\nu_{\tau}$-nucleon inelastic scattering [9], since there is no particle shower at the $\tau$ production vertex, i.e. the first "bang" is missing in our case. If the $\tau$ production occurs outside the detector, but the decay inside it, the process will be indistinguishable from the $\nu_{\tau}$-nucleon deep inelastic scattering background. Another possible source for background is the Glashow resonance itself. It can produce high numbers of $\tau$ (as well as $\mu$ and electron) events, but this is dominant at lower energies where $\Delta^{-}$production is negligible according to the limits $(2)$. Thus, with a fair energy resolution, the Glashow resonance should be distinguishable from the $\Delta^{-}$-resonance.

If one can detect the $\tau$ production vertex without a hadronic shower ("bangless" vertex) and $\tau$ decay after the decay length of the order of some kilometers, one may measure the lepton energy and hence also estimate the neutrino energy. In that case the most serious background for the processes $\Delta^{-} \rightarrow \tau \nu_{\tau}$ are muon neutrino events and cosmic ray muons, with a catastrophic energy loss of the muon after around the same flight distance like $\tau$. The energy loss may be e.g. due to hitting a nucleus in the atmosphere, mimicking the $\tau$ decay. The difference in the signature of muon catastrophic energy loss should be simulated for different detectors, but it is beyond the scope of this letter. Nevertheless, one should be able to statistically distinguish the big sample of decaying $\tau$-leptons, having definite energy and distance from the production vertex, compared to the randomly distributed muon events.

In the following event rate calculations we use as our reference the active galactic nuclei (AGN) models described in [12]. The background calculations are performed in [6]. None of these AGN models have been tested with actual data. Nevertheless, we emphasize the potential importance of observing the high energy neutrino flux and looking at possible resonance effects, which are independent of neutrino sources or the astrophysical models describing neutrino production.

We are interested here in close to horizontally incoming particles. The event rate of the horizontal air showers in a neutrino detector with an area $A$ is

$$
\text { Rate }=A \times t \times \int d E_{\nu} \frac{d N}{d E_{\nu}} \times \sigma\left(E_{\nu}\right) \rho_{e}(h) d h,
$$

where $t$ denotes the time and $\rho_{e}(h)$ is the electron density, calculated from the American Standard Atmosphere [13], depending on the height $h$ from the surface of the Earth. The yearly event rate per steradian is shown in table 1. We have done our estimates for each different AGN model with two different energy resolutions of the detector with a surface area of $10^{6} \mathrm{~km}^{2}$. The first column corresponds to the energy resolution of $25 \%$ (or $2.5 \times 10^{7} \mathrm{GeV}$ ) and the second column that of $5 \%$ (or $5 \times 10^{6} \mathrm{GeV}$ ). We consider the first energy resolution plausible, while the second one is probably optimistic [14]. The event rates have been calculated in each case for two situations. On the first line it is assumed that all three neutrinos have the coupling strenght of $h_{e e}=h_{\mu \mu}=h_{\tau \tau}=1$, corresponding to the decay width $\Gamma\left(\nu_{e} e^{-} \rightarrow \nu_{\ell} \ell^{-}\right)=34.5 \mathrm{GeV}$, while on the second line it is assumed that $h_{e e}=1$ and $h_{\mu \mu} \simeq h_{\tau \tau} \simeq 0$, corresponding to the width $11.5 \mathrm{GeV}$. The increase of events in the case of low energy resolution and large width is perhaps hard to detect. In the case when only the electron neutrino has a large coupling with $\Delta^{-}$one has better chances to see the increase. The best channel for detecting the effect may be $\Delta^{-} \rightarrow \tau \nu_{\tau}$. If one can distinguish the production vertex of $\tau$ to be without hadronic shower (without the first bang), there will be plenty of events per year to be seen. 


\begin{tabular}{c|rr|rr|rr}
$\Gamma_{\Delta}[\mathrm{GeV}]$ & \multicolumn{2}{|c|}{ AGN P96 } & \multicolumn{2}{|c|}{ AGN SS91 } & \multicolumn{2}{|c}{ AGN } \\
\hline \hline 34.5 & 606 & 230 & 207 & 75 & 229 & 88 \\
11.5 & 2230 & 1484 & 740 & 482 & 847 & 566 \\
\hline$\nu N+\bar{\nu} N$ & 4829 & 919 & 1793 & 300 & 1801 & 350 \\
\hline \hline
\end{tabular}

Table 1: Yearly event rate in an atmospheric detector with a surface area of $10^{6} \mathrm{~km}^{2}$ for energy intervals of $5 \times 10^{7} \mathrm{GeV}$ (left row) and $1 \times 10^{7} \mathrm{GeV}$ (right row) for $m_{\Delta}=300 \mathrm{GeV}$.

Water and ice detectors are build in deep water 15] or in antarctic permanent ice 16] and most probably have a final size of just $1 \mathrm{~km}^{3}$. Thus, assuming $m_{\Delta} \gtrsim 200 \mathrm{GeV}$, the events with $\tau$ leptons in the final state are no longer fully contained in the detector. Consequently the observed $\tau$ 's decaying inside the detector will no longer be distinguishable from $\nu_{\tau}$-nucleon deep inelastic scatterings. Signatures of the muons or $\tau$-leptons that are produced inside the detector but that leave it are probably as well inseparable from each other. Events with electrons in the final state might give a signal with long detection times. The event rates per year under these conditions are shown in table 2. 1.

\begin{tabular}{c|rr|rr|rr}
$\Gamma_{\Delta}[\mathrm{GeV}]$ & \multicolumn{2}{|c|}{ AGN P96 } & \multicolumn{2}{|c|}{ AGN SS91 } & \multicolumn{2}{|c}{ AGN M95 } \\
\hline \hline 34.5 & 4.0 & 1.4 & 1.3 & 0.5 & 1.4 & 0.5 \\
11.5 & 14 & 9 & 4.6 & 3.0 & 5.3 & 3.5 \\
\hline$\nu N+\bar{\nu} N$ & 30 & 6 & 11 & 2 & 11 & 2 \\
\hline \hline
\end{tabular}

Table 2: Events in a cubic kilometer sized ice/water detector for energy intervals of $5 \times 10^{7} \mathrm{GeV}$ (left row) and $1 \times 10^{7} \mathrm{GeV}$ (right row) for $m_{\Delta}=300 \mathrm{GeV}$.

Water and ice based neutrino telescopes are effective if $\tau$ decays inside the detector. Observing fully contained events, we allow a maximum path of $500 \mathrm{~m}$ and additional $250 \mathrm{~m}$ for the observation of the $\tau$-decay. In this case the resonant production of $\Delta^{-}$is supposed to happen while the ultra-high energy neutrino propagates the first $250 \mathrm{~m}$ path inside the detector. The mass range $m_{\Delta} \lesssim 150 \mathrm{GeV}$ can be tested this way in cubic kilometer detectors. Event rates for this case are presented in table 3 for $h_{e e}=0.47, h_{\mu \mu}=0.75$ and $h_{\tau \tau}=1$, which are the largest values allowed by the present bounds (2). Non-observation of $\tau$ events with these rates would set new constraints on the couplings.

\begin{tabular}{c|r|r|r} 
& AGN P96 & AGN SS91 & AGN M95 \\
\hline \hline$\Delta^{-}$-resonance & 8.1 & 16.0 & 1.5 \\
Glashow-resonance & 14.1 & 70.5 & 1.2 \\
\hline$\nu N+\bar{\nu} N$ & 10.7 & 21.5 & 1.9 \\
\hline \hline
\end{tabular}

Table 3: Events in a cubic kilometer sized ice/water detector for an energy interval of $1 \times 10^{7} \mathrm{GeV}$ for $m_{\Delta}=150 \mathrm{GeV}$ and $\Gamma_{\Delta}=6.2 \mathrm{GeV}$. In this case it has been assumed that $h_{e e}=0.47, h_{\mu \mu}=0.75$ and $h_{\tau \tau}=1$.

In conclusion, we have investigated the prospects of observing the possible resonant scattering of high-energy cosmic neutrinos on electrons in the atmosphere via an isotriplet scalar exchange. It turn out that it may be, even with large lepton-scalar couplings, hard to distinguish events with muons or electrons in the final state. On the other hand, events with $\tau$-leptons in the final state have an almost backgroundless signature provided it can be checked for each event that there is

\footnotetext{
${ }^{1}$ Note that $\rho_{e(i c e)}=3 \times 10^{29} \mathrm{~m}^{-3}$ and that the detector can work almost $100 \%$ a year. Moreover we assume that a total steradian of $2 \pi$ can be covered.
} 
no hadronic shower in the production vertex of the $\tau$. In large atmospheric arrays, of the size of the OWL [17], one can expect to see of the order of a thousand such events per year.

Acknowledgements. We would like to thank Robert Stokstad and Enrique Zas for helpful discussions. J.M. wishes to thank the Centro de Física Nuclear de Universidade de Lisboa for hospitality. P.K. wishes to thank the Lawrence-Berkeley National Laboratory for hospitality during the final stages of this work and the Jenny and Antti Wihuri foundation and the Finnish Academy of Sciences and Letters for grants. L.B. and P.K. are supported by JNICT under contracts No. BPD.16372 and No. BPD.20182. This work has been supported by the Academy of Finland under the project no. 40677.

\section{References}

[1] S. Glashow, Phys. Rev. 118 (1960) 316.

[2] See e.g., N.G. Deshpande, J.F. Gunion, B. Kayser, Fredrick Olness, Phys. Rev. D44 (1991) 837; J.F. Gunion, Int. J. Mod. Phys. A11 (1996) 1551; Frank Cuypers, Martti Raidal, Nucl. Phys. B501 (1997) 3.

[3] M. Gell-Mann, P. Ramond and R. Slansky, in Supergravity, eds. P. van Niewenhuizen and D. Z. Freedman (North Holland 1979); T. Yanagida, in Proceedings of Workshop on Unified Theory and Baryon Number in the Universe, eds. O. Sawada and A. Sugamoto (KEK 1979); R.N. Mohapatra and G. Senjanovic, Phys. rev. Lett.44 (1980) 912.

[4] M.L. Swartz, Phys. Rev. D 40 (1989) 1521;

M. Lusignoli and S. Petrarca, Phys. Lett. B 226 (1989) 397;

R. Mohapatra, Phys. Rev. D 46 (1992) 2990.

[5] J.A. Grifols, A. Mendez, G.A. Schuler, Mod. Phys. Lett. A4 (1989) 1485 ; J.F. Gunion, Int. J. Mod. Phys. A11 (1996) 1551 ; K. Huitu, J. Maalampi, A. Pietilä, M. Raidal, Nucl. Phys. B 487 (1997) 27.

[6] R. Gandhi, C. Quigg, M.H. Reno and I. Sarcevic, Phys. Rev. D58 (1998) 093009 ; R. Gandhi, C. Quigg, M. H. Reno and I. Sarcevic, Astropart. Phys. 5 (1996) 81.

[7] See e.g. H. Sobel, talk at Neutrino 2000 conference, Sudbury, Canada, June 2000.

[8] See e.g. Y. Suzuki, talk at Neutrino 2000 conference, Sudbury, Canada, June 2000.

[9] J. G. Learned and S. Pakvasa, Astropart. Phys. 3 (1995) 267.

[10] For recent works, see e.g. L. Bento, P. Keränen and J. Maalampi, Phys. Lett. B476 (2000) 205 ;

H. Athar, M. Jezabek and O. Yasuda, TMUP-HEL-0006, hep-ph/0005104.

[11] C. Giunti, M.C. Gonzalez-Garcia and C. Pena-Garay, Phys.Rev. D62 (2000) 013005;

M.C. Gonzalez-Garcia and C. Pena-Garay, IFIC-00-50, hep-ph/0009041;

E. Lisi, talk at Neutrino 2000 conference, Sudbury, Canada, June 2000.

[12] For AGN P96, see R.J. Protheroe, "High-Energy Neutrinos from Blazars" astro-ph/9607165 in Accretion Phenomena and Related Outflows, IAU Colloquium 163, Volume 121 of the ASP Conference Series, ed. D.T. Wickramasinghe, G.V. Bicknell, and L. Ferrario (Astronomical Society of the Pacific, San Francisco, 1997);

For AGN M95, see K. Mannheim, Astropart.Phys. 3 (1995) 295;

For AGN SS91, see F.W. Stecker, C. Done, M.H. Salamon and P. Sommers, Phys.Rev.Lett. 66 (1991) 2697; F.W. Stecker and M.H. Salamon, Space Sci. Rev. 75 (1996) 341, astro-ph/9501064. 
[13] U.S. Standard Atmosphere, 1976, U.S. Government Printing Office, Washington, D.C., 1976.

[14] E. Zas, private communication.

[15] See, NESTOR http://www.roma1.infn.it/nestor/;

NEMO, http://axplns.lns.infn.it/neutrini/;

ANTARES, http://infodan.in2p3.fr/antares,

BAIKAL, http://www.ifh.de/baikal/baikal-home.htm,

[16] See, AMANDA http://amanda.berkely.edu.

[17] See, The OWL Projecthttp://lheawww.gsfc.nasa.gov/docs

gamcosray/hecr/owl_new.html. 